\section{ISSE 2012, Brüssel, 23./24.10.2012}

Die 14. „Information Security Solutions Europe Conference“ (ISSE), ein Gemeinschaftsprojekt der eema (European Association for eIdentity and Security) mit TeleTrusT, findet am 23. und 24.10.2012 in Brüssel statt, um erneut internationale Experten aus Forschung und Wirtschaft zum fachlichen Austausch über aktuelle IT-Sicherheitsthemen, IT-Sicherheitsszenarien, Studienergebnisse und Verfahren zusammenzuführen.

Der Call for Contributions lädt zur Einreichung von Beiträgen bis zum 11.05.2012 ein. Für Informationen siehe http://www.teletrust. de/veranstaltungen/isse/isse-2012/

\section{Offene Türen für Cyber-Kriminelle}

Unternehmen gehen zu nachlässig mit der Sicherheit ihrer Kundendaten um, zeigt eine am 06.03.2012 veröffentlichte Studie der Unternehmensberatung A.T. Kearney.

Die Investitionen in die IT-Sicherheit halten demnach nicht Schritt mit dem Anstieg der Bedrohung. Vor allem im Online-Geschäft sind Datendiebstähle an der Tagesordnung. Auch in Deutschland: Dort liegt die Internetkriminalität auf Rekordniveau. Die Kunden verlieren daher das Vertrauen in die Unternehmen. Wer sich nur ungenügend um das Thema Sicherheit kümmert, riskiert somit, vom lukrativen Internet-Markt ausgeschlossen zu werden.

In den vergangenen Jahren haben die Investitionen der Firmen in die Datensicherheit um gerade mal 21 Prozent zugenommen, so die Studie. „Das ist zu wenig, wenn man bedenkt, dass zum Beispiel in den USA gleichzeitig die Anzahl von Internet-Straftaten um ungefähr 300 Prozent angestiegen ist", sagt Dr. Marcus Eul, Partner bei A.T. Kearney in Düsseldorf. Das Problem stellt sich auch deutschen Unternehmen: 2010 wurden hierzulande so viele Straftaten über das Internet begangen, wie noch nie zuvor. Insgesamt waren es laut Polizeilicher Kriminalstatistik rund 223.000 Fälle, bei 182.000 handelte es sich um Betrugsdelikte.

Ein Grund für die mangelnde Sensibilität könne sein, dass der Raub von Daten häufig nicht direkt dem Unternehmen, sondern zunächst nur dem Kunden schadet, glaubt Eul. So zeigt die Untersuchung, dass zum Beispiel bei Identitätsdiebstahl der durchschnittliche Schaden pro Opfer zwischen 1.900 und 4.800 US-Dollar liegt. In den USA war bereits jeder zehnte Konsument von einem solchen Identitätsdiebstahl betroffen. Dabei entwenden Cyber-Kriminelle die Kundendaten, um diese für eigene Einkäufe oder weitere Straftaten zu missbrauchen.

Firmen, die sich nur unzureichend um ihre IT-Sicherheit kümmern, schaden aber auch sich selbst. „Spektakuläre Online-Attacken wie etwa gegen Sony oder die Citibank haben die Kunden verunsichert", meint Eul. „Viele fragen sich, ob ihre Daten überhaupt noch irgendwo sicher sind." Vertrauen ist jedoch die wichtigste Stütze des Internet-Handels. Wenn Firmen den Schutz ihrer Daten ignorieren, untergraben sie somit die Grundlagen des eigenen Online-Geschäfts.

Die Studie zeigt weiterhin: Datendiebstähle können für Unternehmen fatale Folgen haben. Der Wert des Unternehmens Sony sank von Januar 2011 bis Januar 2012 um 51 Prozent. Der Elektronikkonzern war bereits 13 mal Ziel von Hacker-Angriffen. Allein im vergangenen Jahr gab es mehrere Attacken. Experten schätzen die Höhe des Schadens auf 170 Millionen US-Dollar - zuzüglich der langfristigen Rufschädigung.

Besonders die IT-Verantwortlichen in den Firmen sind nun gefordert. „Sie müssen für die notwendige Datensicherheit zu sorgen, um die Kunden, das Unternehmen und letztlich auch den eigenen Arbeitsplatz zu schützen", so Eul. Es gebe bereits $\mathrm{CIO}$, die wegen Sicherheitsproblemen ihren Job verloren haben.

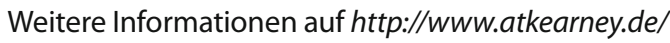

\title{
Rezensionen
}

\section{Veranstaltungen}

\section{RSA Conference, 27.02. - 02.03.2012 in San Francisco, USA}

Die weltgrößte IT-Sicherheitskonferenz fand auch diesmal wieder im Moscone Center in San Francisco statt. Die RSA Konferenz ist nach wie vor die Welt-Leit-Messe für IT-Security mit starker internationaler Beteiligung. Mit ca. 16.000 Konferenzteilnehmern und etwa 350 Ausstellern ist diese Veranstaltung an den Grenzen der Kapazitäten des Moscone Centers North und South angekommen. Da weiteres Wachstum zu erwarten ist, soll ab 2014 dieses Gelände mit beiden großen Hallen für die Ausstellung zur Verfügung stehen und zusätzlich das Moscone Center West für die Keynote Präsentationen genutzt werden. Besaß die Veranstaltung früher eindeutig den Charakter einer Fachkonferenz mit begleitender Ausstellung, erreicht die Messe inzwischen prägenden Einfluß.

,IT Security Made in Germany' war zum 12. Mal in ununterbrochener Folge, mit einer vom Bundesministerium für Wirtschaft und Technologie (BMWi) und vom Ausstellungs- und Messe-Ausschuss der deutschen Wirtschaft e.V. (AUMA) geförderten und von Tele-
TrusT organisierten Gemeinschaftsausstellung vertreten. 17 deutsche Unternehmen und das Bundesamt für Sicherheit in der Informationstechnik (BSI) vermittelten in einem Informationszentrum ein überzeugendes Bild von der Kompetenz und Wettbewerbsfähigkeit der deutschen IT-Sicherheitsbranche. Mit $140 \mathrm{~m}^{2}$ Standfläche in der Hauptachse der Ausstellung war eine gute Sichtbarkeit der deutschen Präsenz gegeben. Allerdings ist festzustellen, dass die Standgestaltung anderer Aussteller zunehmend aufwendiger wird und damit die relativ statische Optik des deutschen Auftritts an Attraktivität verliert. Durch den Status eines Goldsponsors für ,IT Security Made in Germany' ist es jedoch gegeben, dass die Konferenzteilnehmer zu vielen Gelegenheiten (Key Note Sessions, Konferenzbroschüre, Übersicht über das deutsche Angebot in den Konferenztaschen aller Teilnehmer) auf die deutsche Beteiligung aufmerksam gemacht wurden.

Einen hohen Stellenwert besitzt auch das von TeleTrusT mit den am Gemeinschaftsauftritt Beteiligten gestaltete Rahmenprogramm. Es war in diesem Jahr durch die aktive Mitwirkung der Staatssekretärin im Ministerium des Innern und Bundes $\mathrm{CIO}$, Cornelia Rogall-Grothe, besonders erfolgreich. 